\title{
Actividad antifúngica in vitro de diferentes concentraciones de Syzygium aromaticum "clavo de olor" contra Fusarium oxysporum
}

\section{In vitro antifungal activity of different variations of Syzygium aromaticum "clavo de olor" against Fusarium oxysporum}

\author{
Victor John Acedo-Zegarra ${ }^{1 *}$ (D), Diego Fernando Arana-Bardales ${ }^{1}$, Alberto Enrique Condo-Montano ${ }^{1}$
}

\begin{abstract}
RESUMEN
Se determinó la actividad antifúngica de Syzygium aromaticum "clavo de olor" a concentraciones de 1\%, 3\% y 5\% contra el hongo fitopatógeno Fusarium oxysporum. Para ello se realizaron cultivos in vitro de dicho hongo, estableciendo dos placas Petri control y tres repeticiones para cada una de las concentraciones: $1 \%, 3 \%$ y $5 \%$ de Syzygium aromaticum. El crecimiento radial de las colonias se evaluó durante cinco días, midiendo el radio de las mismas con un vernier. Como resultados se obtuvo que el crecimiento radial del micelio de F. oxysporum para los controles, así como para las concentraciones de $1 \%$ y 3\% de S. aromaticum son de $2.65 \mathrm{~cm}, 2.5 \mathrm{~cm}$ y $1.1 \mathrm{~cm}$, respectivamente, considerándose los datos más altos de crecimiento micelial en estas placas. Además, para la concentración de 5\% de S. aromaticum, no se observa crecimiento micelial del hongo. Así mismo, en relación a las concentraciones de $1 \%$ y $3 \%$ de S. aromaticum, el porcentaje de inhibición en relación al crecimiento micelial de $\mathrm{F}$. oxysporum es de $11.88 \%$ y $65.45 \%$, respectivamente, y para la concentración de $5 \%$ de extracto de Syzygium aromaticum los resultados obtenidos muestran notablemente una actividad antifúngica total de $100 \%$ frente a Fusarium oxysporum.
\end{abstract}

Palabras claves: extracto, actividad antifúngica, hongo fitopatógeno, clavo de olor (Syzygium aromaticum).

\begin{abstract}
The antifungal activity of Syzygium aromaticum "clove" was determined at concentrations of 1\%, 3\% and 5\% against the phytopathogenic fungus Fusarium oxysporum. For this, in vitro cultures of said fungus were carried out, establishing two control Petri dishes and three repetitions for each of the concentrations: $1 \%, 3 \%$ and 5\% of Syzygium aromaticum. The radial growth of the colonies was evaluated for five days, measuring their radius with a vernier. As a result, it was obtained that the radial growth of the mycelium of $F$. oxysporum for the controls as well as for the concentrations of $1 \%$ and $3 \%$ of $S$. aromaticum are $2.65 \mathrm{~cm}, 2.5 \mathrm{~cm}$ and $1.1 \mathrm{~cm}$, respectively, considering the highest data of mycelial growth in these plates. In addition, for the $5 \%$ concentration of S. aromaticum, no fungal mycelial growth is observed. Likewise, in relation to the concentrations of $1 \%$ and $3 \%$ of $S$. aromaticum, the percentage of inhibition in relation to the mycelial growth of $F$. oxysporum is $11.88 \%$ and $65.45 \%$, respectively, and for the concentration of $5 \%$ of Syzygium aromaticum extract the results obtained remarkably show a total antifungal activity of $100 \%$ against $F$ usarium oxysporum.
\end{abstract}

Keywords: extract, antifungal activity, plant pathogenic fungus, clove (Syzygium aromaticum).

\footnotetext{
${ }^{1}$ Universidad Nacional de Trujillo, Trujillo, Perú

"Autor de Correspondencia, e-mail:johnacedo209@gmail.com
} 


\section{INTRODUCCIÓN}

La población mundial hoy en día está en incremento y con ello, ha aumentado la necesidad por contar con una mayor producción de alimentos, con la finalidad de satisfacer esta necesidad en la población. Debido a ello, el hombre y su interés por obtener grandes producciones agrícolas, además de su protección, ha optado por emplear indiscriminadamente, y muchas veces inconscientemente, los plaguicidas químicos (Palacios et al., 2011).

Actualmente, los sistemas agrícolas en búsqueda de cumplir con esta necesidad de obtener una gran producción y de calidad, también se han visto afectados por el uso de agroquímicos para controlar el ataque de malezas, plagas y enfermedades que atacan a los diferentes cultivos y ello ha generado diversos problemas no sólo sobre la salud humana sino también a nivel ambiental (Gan y Wickings, 2017).

Sin embargo, este tipo de agricultura cada día es más insostenible e irresponsable debido al empleo de prácticas y manejos con enfoques buscando la sostenibilidad de la producción, debido a las exigencias que plantean los mercados diferenciados, los cuales a través de sus organismos reguladores hacen cumplir estándares de producción específicos. Además, para el cumplimiento de estas exigencias, las prácticas empleadas se están basando en el uso de productos químicos lo cual está creando resistencia por parte de los microorganismos a los plaguicidas; por ello, las industrias están buscando diferentes alternativas de manejo responsable (Gavrilescu y Chisti, 2005; Shahid et al., 2017).

Por ejemplo, cultivos de hortalizas y frutales están siendo tratados contra enfermedades como alternaria (Alternaria solani), fusarium (Fusarium oxysporum) y antracnosis (Colletotrichum gloesporioides), principalmente con el uso de plaguicidas de síntesis química. Además de esta práctica realizada en campo, algunos productores también aplican productos químicos a sus productos post cosecha, con la finalidad de mantenerlos sin presencia de patógenos para la venta, por lo que estos productos no logran degradarse, provocando la reducción de la calidad biológica en los productos a comercializar (Ramirez et al., 2016).

Sin embargo, en los últimos años el uso de estos productos químicos o fungicidas de composición química, ha aumentado la preocupación no sólo del consumidor sino también del productor. Esto ha generado que el uso de estos productos sea cada vez más restrictivo debido a las consecuencias que provoca tanto en la salud, como efectos carcinógenos, problemas de toxicidad residual, como también en la contaminación ambiental provocando la disminución de biodiversidad, contaminación del suelo y aparición de resistencia microbiana (Mesa et al., 2019).

Debido a ello, en estos últimos años, se han buscado alternativas de producción de calidad y principalmente sostenible, en donde se obtenga una producción de libre de compuestos químicos que dañen a las personas o al medio ambiente, encontrando como una gran opción el uso de controladores biológicos y compuestos orgánicos. Esto se debe a que las especies vegetales tienen compuestos que cumplen funciones biológicas y químicas de defensa, por lo que gran variedad de estos elementos o compuestos pueden tener actividad biológica sobre los microorganismos, dentro de ellos los hongos (Mazid et al., 2011; Saravanakumar et al., 2015).

Los elementos y compuestos que proporcionan a las plantas propiedades antifúngicas para enfrentar diferentes patógenos, pueden actuar de individualmente, así como también mediante mezclas a diferentes concentraciones y/o proporciones; así mismo, existen diferentes métodos para la obtención o extracción de estos compuestos los cuales buscan salvaguardar las propiedades extraídas de la mejor manera posible (Villa-Martínez et al., 2015).

Las especies vegetales y los extractos obtenidos de ellos, en los últimos años, han sido de gran interés científico para la búsqueda de nuevas investigaciones en relación a su actividad antibacteriana y antifúngica y, además, de nuevas propiedades de las mismas (Briceño et al., 2011). Además, estas propiedades con las que cuentan las especies vegetales es gracias a sus 
extractos y aceites vegetales presentes, ya que estos contienen grupos químicos y componentes activos que actúan sobre la resistencia, repelencia y control total de microorganismos e insectos, tales como terpenos, fenoles alcaloides, ácidos orgánicos, péptidos, ácidos grasos polinsaturados y del grupo Omega 3, Piperina, Capsicina, D - Limonene, diatomos, cafeína, nicotina, entre otros (Corrales et al., 2018).

Por ello, surge la alternativa de usar los extractos obtenidos de especies vegetales para el control de plagas y enfermedades, con la finalidad de lograr una agricultura sostenible, debido a la elevada efectividad de acción, bajo costo y no provocar contaminación al ambiente (Rodríguez et al., 2000). Dentro de estas especies vegetales que cuentan con estas características tenemos a Syzygium aromaticum "clavo de olor", a la cual se le atribuyen propiedades antifúngicas, antibacterianas y otros.

Syzygium aromaticum es una especie de fácil localización y es de gran comercialización debido a sus propiedades antifúngicas, antisépticas, antivirales, anestésicas analgésicas, bactericidas, atribuyendo estas propiedades al componente eugenol, quien también es el responsable de la mayor parte del aroma característico de la especie, comprendiendo entre el $72-90 \%$ del aceite esencial extraído principalmente de los botones (Cueva, 2017).

Por otro lado, entre los hongos y oomycetos fitopatógenos más significativos tanto en pre como en poscosecha de los distintos cultivos se encuentran algunos géneros como Botrytis, Puccinia, Rhizoctonia, Cladosporium, Fusarium, Colletotrichum, Aspergillus, Penicillium, Alternaria, Mycosphaerella, Ceratocystis, Sphaerotheca, Phytophthora, entre otros (Hosni et al., 2013; Castaño-Zapata, 2015). Dentro de uno de este grupo de hongos fitopatógenos, el más conocido es el género Fusarium, debido a su elevada capacidad fitopatógena que afecta a una gran variedad de cultivos (Forero-Reyes et al., 2018).

El género Fusarium pertenecen a un grupo de hongos ascomicetos filamentosos y cosmopolitas, los cuales presentan un micelio bien desarrollado, septado y conidióforos característicos, aunque algunas especies tienen un talo unicelular; además, los hongos de este género son considerados principalmente como hongos de campo, debido a que actúan sobre diversos cultivos y les causa una diversidad de enfermedades (Sumalan et al., 2013). Por ello, las especies de Fusarium son ampliamente conocidos alrededor del mundo, y se han convertido en un problema serio ya que producen metabolitos tóxicos que afectan a la biodiversidad, debido a la gran cantidad de enfermedades que afectan a los cultivos de todo el mundo, generando además importantes pérdidas económicas para la población (Villa-Martínez et al., 2015).

En el presente trabajo se determinó la actividad antifúngica in vitro de diferentes concentraciones de Syzygium aromaticum "clavo de olor" contra Fusarium oxysporum, para la inhibición de este hongo, considerado como uno de los principales hongos fitopatógenos que ataca a diversos cultivos.

\section{MATERIALY MÉTODOS}

\section{Material Biológico}

La colecta de los botones florales de $S$. aromaticum "clavo de olor", fueron obtenidos en el mercado Mayorista de la ciudad de Trujillo. Posteriormente las muestras recolectadas fueron trasladadas al Laboratorio de Fitopatología de la Facultad de Ciencias Biológicas de la Universidad Nacional de Trujillo, las cuales fueron seleccionadas desechándose las que presentaron daños mecánicos.

Las cepas utilizadas del hongo fitopatógeno, F. oxysporum, fueron proporcionadas por la Cátedra de Fitopatología de la Facultad de Ciencias Biológicas de la misma universidad.

\section{Obtención del extracto}

Los botones florales de $S$. aromaticum fueron molidos en un "molino casero".

Luego, se pesaron $25 \mathrm{~g}$ de cada muestra adicionándole $100 \mathrm{~mL}$ de disolvente, agua destilada, en un matraz para obtener una solución al 25\%, y se dejó reposar durante $48 \mathrm{~h}$ a temperatura ambiente. Posteriormente se filtró el extracto con papel filtro Whatman $\mathrm{N}^{\circ} 1$. 
A partir de esta concentración (25\%) se realizaron diluciones al 1, 3 y 5\% con vistas a determinar la concentración de $S$. sativum "clavo de olor", a la cual el crecimiento micelial del hongo $F$. oxysporum se inhibe.

Se realizaron pruebas in vitro con medio de cultivo artificial papa dextrosa agar (PDA), adicionando los $\mathrm{mL}$ correspondientes de las diluciones de S. sativum (Tabla 1), con tres repeticiones para cada concentración.

Tabla 1. Volúmenes correspondientes de diluciones de Syzygium aromaticum "clavo de olor" para la evaluación antifúngica contra Fusarium oxysporum.

\begin{tabular}{cccc}
\hline$\%$ & $\begin{array}{c}\text { Volumen } \\
\text { inicial }\end{array}$ & C1 & $\begin{array}{c}\text { Volumen } \\
\text { dilución }\end{array}$ \\
\hline $1 \%$ & $100 \mathrm{~mL}$ & $25 \%$ & $4 \mathrm{~mL}$ \\
$3 \%$ & $100 \mathrm{~mL}$ & $25 \%$ & $12 \mathrm{~mL}$ \\
$5 \%$ & $100 \mathrm{~mL}$ & $25 \%$ & $20 \mathrm{~mL}$ \\
\hline
\end{tabular}

\section{Evaluación de la actividad antifúngica}

Se procedió a realizar la esterilización de las concentraciones de $S$. aromaticum, los cuales fueron esterilizados a $121^{\circ} \mathrm{C}$. Luego, el medio de cultivo PDA conteniendo las concentraciones de $S$. aromaticum fueron vaciados en placas de petri $(60 \times 15 \mathrm{~mm})$ para su solidificación. Se usaron tres repeticiones para cada una de las concentraciones. Las placas de Petri control contenían únicamente PDA.

Posteriormente, se realizó la siembra de $F$. oxysporum en cada una de las placas de estudio. Con un asa bacteriológica, esterilizada, se tomó una cantidad de inóculo del hongo y se introdujo en el centro del medio de cultivo que contiene la placa petri. Las placas fueron incubadas en la oscuridad por un día a $22^{\circ} \mathrm{C}$ y el crecimiento radial del micelio se midió en $\mathrm{cm}$ con un vernier durante 5 días. Todos los bioensayos fueron llevados a cabo por triplicado.

\section{Análisis de datos}

Al finalizar la evaluación, se realizó el análisis de datos calculando el porcentaje de inhibición. El efecto inhibitorio de los extractos se determinó como porcentaje de inhibición del crecimiento radial del micelio de las especies de los hongos con respecto al testigo PDA.

Para ello, este efecto inhibitorio del crecimiento micelial se calculó de la siguiente manera: \% inhibición = [(crecimiento micelial del testigo - crecimiento micelial del tratamiento) / crecimiento micelial del testigo] $\mathrm{x} 100$.

Los resultados obtenidos se evaluaron estadísticamente, mediante un Análisis de Varianza (ANOVA) para comparar las diferentes concentraciones de $S$. aromaticum "clavo de olor", con la finalidad de determinar qué concentración es el que tiene mayor actividad antifúngica frente al hongo fitopatógeno $F$. oxysporum.

\section{RESULTADOS}

En la tabla 2 se presentan los resultados obtenidos del crecimiento radial del micelio del hongo fitopatógeno F. oxysporum expuestos a concentraciones de $S$. aromaticum "clavo de olor", evaluados durante cinco días. En esta tabla se observa que el crecimiento radial del micelio (cm) de F. oxysporum en los controles va en aumento conforme pasan los días. Así mismo, para las concentraciones de $1 \%$ y $3 \%$ de $S$. aromaticum, el crecimiento de F. oxysporum también aumenta conforme pasan los días. Sin embargo, este hongo no presenta crecimiento micelial a una concentración de $5 \%$ de S. aromaticum.

La figura 1 representa el crecimiento micelial a los cinco días de sembrado del hongo F. oxysporum, para cada una de las concentraciones empleadas de S. aromaticum $(1 \%, 3 \%$ y $5 \%)$ con sus respectivas repeticiones y los controles empleados.

La tabla 3 y la tabla 4 presentan el porcentaje de inhibición de $S$. aromaticum, a las concentraciones de $1 \%$, $3 \%$ y $5 \%$ frente al hongo fitopatógeno $F$. oxysporum y el promedio de estos porcentajes de $S$. aromaticum frente a $F$. oxysporum, respectivamente. Se puede observar claramente que con una concentración de $5 \%$ de $S$. aromaticum se obtiene una inhibición total del $100 \%$ en relación al crecimiento micelial del hongo fitopatógeno $F$. oxysporum, lo cual indica una propiedad antifúngica muy efectiva. 
Tabla 2. Datos del crecimiento radial del micelio (cm) de F. oxysporum a concentraciones de $1 \%$, 3\% y $5 \%$ de $S$. aromaticum, evaluados durante cinco días

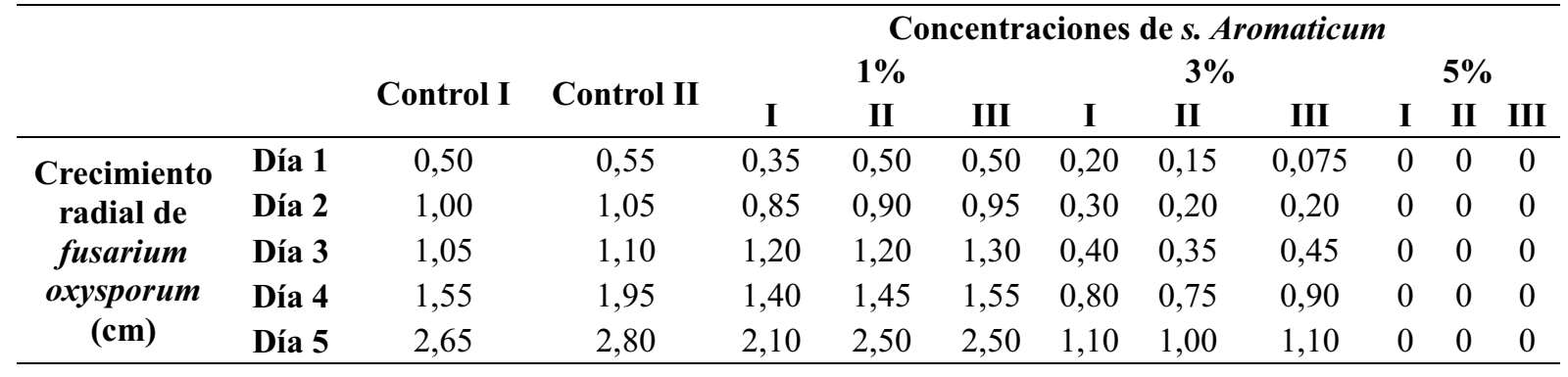

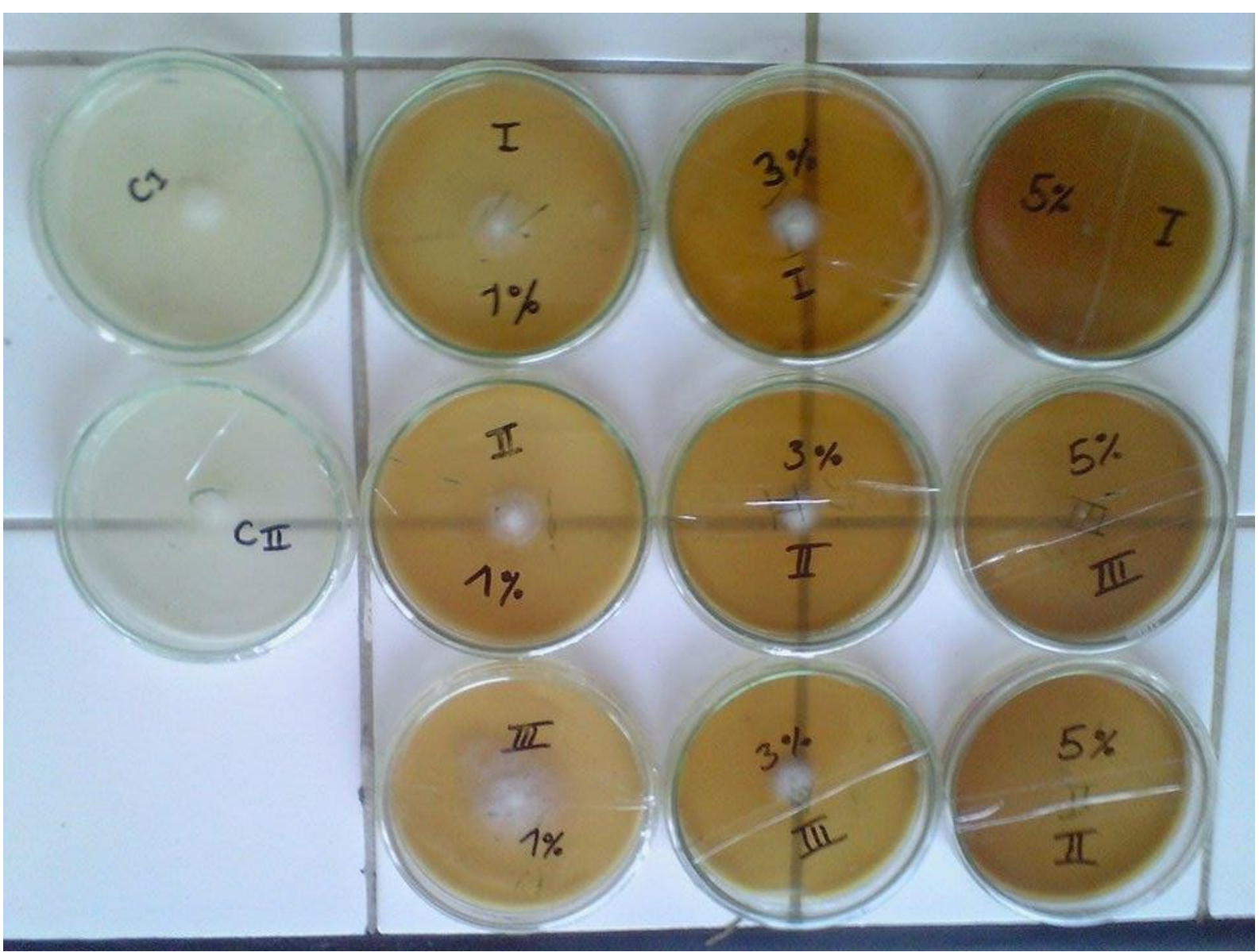

Figura 1. Crecimiento micelial de F. oxysporum a los cinco días de sembrado, para cada concentración de $S$. aromaticum, con sus respectivas repeticiones.

Tabla 3. Porcentaje de inhibición de S. aromaticum "clavo de olor" a las concentraciones de $1 \%, 3 \%$ y $5 \%$ frente al hongo fitopatógeno $F$. oxysporum

\begin{tabular}{|c|c|c|c|c|c|c|c|c|c|}
\hline & \multicolumn{9}{|c|}{ Porcentaje de inhibición } \\
\hline & \multicolumn{3}{|c|}{$1 \%$} & \multicolumn{3}{|c|}{$3 \%$} & \multicolumn{3}{|c|}{$5 \%$} \\
\hline & I & II & III & I & II & III & I & II & III \\
\hline Día 1 & 33,33 & 4,76 & 4,76 & 61,9 & 71,43 & 85,71 & 100 & 100 & 100 \\
\hline Día 2 & 17,07 & 12,20 & 7,32 & 70,73 & 80,49 & 80,49 & 100 & 100 & 100 \\
\hline Día 3 & 4,19 & 4,19 & 2,33 & 62,79 & 67,44 & 58,14 & 100 & 100 & 100 \\
\hline Día 4 & 20,00 & 17,10 & 11,4 & 54,30 & 57,10 & 48,60 & 100 & 100 & 100 \\
\hline Día 5 & 22,94 & 8,26 & 8,26 & 59,63 & 63,30 & 59,63 & 100 & 100 & 100 \\
\hline
\end{tabular}


Tabla 4. Promedio de porcentaje de inhibición de S. aromaticum "clavo de olor" a las concentraciones para cada concentración (1\%, 3\% y 5\%) frente al Hongo Fitopatógeno Fusarium oxysporum

\begin{tabular}{|c|c|c|c|}
\hline & \multicolumn{3}{|c|}{ Porcentaje de inhibición } \\
\hline & $1 \%$ & $3 \%$ & $5 \%$ \\
\hline Día 1 & 14,286 & 73,016 & 100 \\
\hline Día 2 & 12,195 & 77,236 & 100 \\
\hline Día 3 & 3,566 & 62,791 & 100 \\
\hline Día 4 & 16,190 & 53,333 & 100 \\
\hline Día 5 & 13,150 & 60,856 & 100 \\
\hline Promedio \% inhibición por concentración & 11,877 & 65,446 & 100 \\
\hline
\end{tabular}

Estos resultados nos permitieron realizar un análisis ANOVA en relación a los niveles de concentración empleados para el estudio, en donde se puede demostrar que existe suficiente base estadística para afirmar que, para las tres concentraciones de $S$. aromaticum “clavo de olor", sí existen diferencias significativas respecto a la actividad antifúngica de cada una de ellas frente al hongo fitopatógeno $F$. oxysporum, con un porcentaje de error de $5 \%$ (Tabla 5).

Tabla 5. Análisis de Varianza (ANOVA) para el carácter: “Concentración” de clavo de olor, Syzygium aromaticum.

\begin{tabular}{cccccc}
\hline *Origen de las & G.L. & S.C. & C.M. & Fc & Ft \\
variaciones & 2 & 19715,30 & 9857,65 & 253,66 & 3,89 \\
Entre grupos & 12 & 466,34 & 38,86 & & \\
Dentro de los grupos & 14 & 20181,64 & & & \\
Total &
\end{tabular}

G.L. $=$ Grados de libertad; S.C. $=$ Suma de cuadrados; C.M. $=$ Cuadrados medios $; \mathrm{Fc}=$ Estadístico F calculado $; \mathrm{Ft}=\mathrm{Estadístico} \mathrm{F} \mathrm{teórico}$

\section{IV.DISCUSIÓN}

El crecimiento radial del micelio de F. oxysporum para los controles, así como para las concentraciones de $1 \%$ y $3 \%$ de S. aromaticum son de $2.65 \mathrm{~cm}, 2.5 \mathrm{~cm}$ y 1.1 $\mathrm{cm}$, respectivamente, considerándose los datos más altos de crecimiento micelial en estas placas. Además, para la concentración de $5 \%$ de $S$. aromaticum, no se observa crecimiento micelial del hongo. Así mismo, en relación a las concentraciones de $1 \%$ y $3 \%$ de $S$. aromaticum, el porcentaje de inhibición en relación al crecimiento micelial de $F$. oxysporum es de $11.88 \%$ y $65.45 \%$ respectivamente.

La evaluación de las concentraciones de $1 \%$ y $3 \%$ presentaron un porcentaje de inhibición menor frente al crecimiento micelial del hongo fitopatógeno Fusarium oxysporum. Además, se puede observar claramente que con una concentración de $5 \%$ de $S$. aromaticum se obtiene una inhibición total del 100\% en relación al crecimiento micelial del hongo fitopatógeno $F$. oxysporum, lo cual indica una propiedad antifúngica muy efectiva.

Los resultados de nuestra investigación coinciden con diversos estudios realizados empleando S. aromati- cum frente al crecimiento del hongo Fusarium. Por ejemplo, Pawar y Thaker (2007) realizaron estudios empleando aceites esenciales para el control de Fusarium oxysporum $\mathrm{f}$. sp. cicer, entre ellos los más eficaces fueron de canela (Cinnamomum zeylanicum), casia (Cinnamomum cassia), clavo de olor (Syzygium aromaticum) y hierba de limón (Cymbopogon citratus), pues tuvieron un alto efecto inhibitorio.

Así mismo, Velluti et al., 2004 en sus estudios evaluaron el crecimiento de especies de hongos, como Fusarium verticillioides, F. proliferatum y F. graminearum, con extractos vegetales y/o aceites esenciales de canela, clavo de olor, orégano, hierba de limón y palmarosa, en donde presentaron inhibición en las especies de Fusarium.

Cardiet et al., 2011, enfrentó otra especie de este hongo, Fusarium graminearum, frente a extractos de Syzygium aromaticum "clavo de olor", donde también se observaron resultados de inhibición en el crecimiento micelial del hongo en mención.

Estudios de Rueda et al. (2013), realizaron estudios de diferentes extractos, dentro de los cuales emplearon el extracto acuoso de Syzygium aromaticum "clavo de 
olor”. En esta evaluación, el extracto presentó actividad biológica para diferentes especies de hongos empleados en su estudio, dentro de los cuales estaba incluido Fusarium oxysporum. Además, estos resultados fueron relacionados a otros en donde también demostraron que el aceite esencial de clavo posee actividad antifúngica in vitro, afectando especies que se desarrollan frecuentemente en los alimentos tales como Paeciliomyces, Peniclilium spp., Rhizophus spp., Rhizomucor spp., inclusive algunas especies de Aspergillus (Joseph and Sujatha, 2011) y que inhibe perceptiblemente el crecimiento de Aspergillus flavus, A. niger, Fusarium oxysporum, F. chrysogenum, Penicillium spp. (Kritzinger et al., 2002).

\section{CONCLUSIONES}

Syzyygium aromaticum "clavo de olor", presenta una actividad antifúngica contra el hongo fitopatógeno Fusarium oxysporum a una concentración de 5\%, presentando propiedades antifúngicas efectivas, con un porcentaje de inhibición de $100 \%$, esto debido al efecto inhibitorio que posee esta especie.

Teniendo en cuenta ello, la investigación realizada servirá como una propuesta para proponer investigaciones aplicativas, con la finalidad de determinar el efecto de estos componentes sobre frutos en estudios in situ.

\section{REFERENCIAS BIBLIOGRÁFICAS}

Briceño, G., J. García, A. Maselli y L.G. Rosales. 2011. "Effect of ethanolic extracts of rue and neem on the control of phytopathogenic bacteria of the genus Erwinia." Agronomía Tropical 61 (2): 141-148.

Cardiet, G., B. Fuzeau, C. Barreau, y F. Fleurat. 2012. "Contact and fumigant toxicity of some essential oil constituents against a grain insect pest Sitophilus oryzae and two fungi, Aspergillus westerdijkiae and Fusarium graminearum." Journal of Pest Science 85 (3):351-358.

Castaño-Zapata, J. 2015. Principios básicos de hon- gos fitopatógenos. Manizales (Colombia): Universidad de Caldas.

Corrales, C. J., A. A. Rodríguez, M. K. Villalobos, V. S. Hernández, y R. O. Alvarado. 2018. "Evaluación de tres extractos naturales contra Bemisia tabaco en el cultivo del melón, Puntarenas, Costa Rica." Agronomía Costarricense 42 (2): 93-106.

Cueva, B., J.A. 2017. Actividad antifúngica in vitro del aceite esencial y extracto alcohólico del Syzygium aromaticum "clavo de olor" sobre Candida albicans CEPA ATCC 10231. Trabajo de Grado. Universidad Nacional de Chimborazo. Riobamba(Ecuador).

Forero-Reyes, C. M., A. M. Alvarado, A. M. Ceballos, L. C. González, M. Y. Linares-Linares, R. Castañeda, A. Pulido, M. Góngora, J. A Cortés-Vecino, y M. X. Rodríguez-Bocanegra. 2018. "Evaluación de la capacidad patogénica de Fusarium spp. en modelos vegetal y murino." Revista Argentina de Microbiología, 50 (1): 90-96.

Gan, H. y K. Wickings. 2017. "Soil ecological responses to pest management in golf turf vary with management intensity, pesticide identity, and application program." Agriculture, Ecosystems \& Environment, 246: 66-77.

Gavrilescu, M. y Y. Chisti. 2005. "Biotechnology-a sustainable alternative for chemical industry." Biotechnology Advances 23 (7): 471-499.

Hosni, K., I. Hassen, H. Sebei, y H. Casabianca. 2013. "Secondary metabolities from Chrysanthemum coronarium (Garland) flowerheads: Chemical composition and biological activities." Industria Crops and Products 44: 263271.

Joseph, B., y S. Sujatha. 2011. "Bioactive compounds and its autochthonous microbial activities of extract and clove oil (Syzygium aromaticum) on some food borne pathogens." Asian Journal of Biological Sciences 4: 35-43.

Kritzinger, Q., T. Aveling, y W. Marasas. 2002. "Effect 
of essential plant oils on storage fungi, germination and emergence of cowpea seeds." Journal of Seed Science and Technology 30: 609-619.

Mesa, V. A. M., P. A. Marín, O. Ocampo, J. Calle, y Z. Monsalve. 2019. "Fungicidas a partir de extractos vegetales: una alternativa en el manejo integrado de hongos fitopatógenos." Revista de Investigaciones Agropecuarias 45 (1).

Mazid, S., J. Kalita, y R. Rajkhowa. 2011. “A review on the use of biopesticides in insect pest management." International Journal of Science and Advanced Technology 1 (7): 169-178.

Palacios, J. R., C. R. Romeu, O. Ramírez, L. Leliebre, y M. Ortiz. 2011. “Actividad antifúngica in vitro de extractos de Navisporus floccosus y Ganoderma sp. contra hongos fitopatógenos." Fitosanidad 15(2): 123-128.

Pawar, V. C. y V. S. Thaker. 2007. "Evaluation of the anti-Fusarium oxysporum $\mathrm{f}$. sp cicer and antiAlternaria porri effects of some essential oils." World Journal of Microbiology and Biotechnology 23 (8): 1099-1106.

Ramírez, S. I., B. O. López, Z. S. Espinosa, y V. Wong. 2016. "Actividad antifúngica de hidrodestilados y aceites sobre Alternaria solani, Fusarium oxysporum y Colletotrichum gloesporioides." Revista Mexicana de Ciencias Agrícolas 7 (8): 1879-1891.

Rodríguez A. T., D. Morales, y M. Ramírez. 2000. "Efecto de extractos vegetales sobre el crecimiento in vitro de hongos fitopatógenos." Cultivos Tropicales 21 (2): 79-82.

Rueda de León, I. C., V. Colorado, M. Salas, C. Muñoz, O. Hernández. 2013. “Actividad Antifúngica in vitro de extractos acuosos de especias contra Fusarium oxysporum, Alternaria alternata, Geotrichum candidum, Trichoderma spp., Penicillum digitatum y Aspergillus niger." Revista Mexicana de Fitopatología 31 (2): 105-112.
Saravanakumar, D., L. Karthiba, R. Ramjegathesh, K. Prabakar, y T. Raguchander. 2015. "Characterization of bioactive compounds from botanicals for the management of plant diseases." En Sustainable Crop Disease Management Using Natural Products. Ganesan, S., K. Vadivel, y J. Jayaraman (eds). Boston (EE.UU.): CAB Internacional.

Shahid, M., A. Zaidi, M. S. Khan, A. Rizvi, S. Saif, y B. Ahmed. 2017. "Recent advances in Management Strategies of Vegetable Diseases." En Microbial Strategies for Vegetable Production. Zaidi, A y M. S. Khan (eds). Nueva York (EE. UU.): Springer International Publishing. DOI 10.1007/978-3-319-544014_9.

Sumalan, R. M., E. Alexa, y M. A. Poiana. 2013. “Assessment of inhibitory potential of essential oils on natural mycoflora and Fusarium mycotoxins production in wheat." Chemistry Central Journal 7(1): 1-12.

Velluti A., S. Marín, P. Gonzalez, A. Ramos, y V. Sanchis. 2004. "Initial screening for inhibitory activity of essential oils on growth of Fusarium verticillioides, $F$. proliferatum and F. graminearum on maize-based agar media." Food microbiology 21 (6): 649-656.

Villa A., R. Pérez, H. Morales, M. Basurto, J. Soto, y E. Martínez. 2015. "Situación actual en el control de Fusarium spp. y evaluación de la actividad antifúngica de extractos vegetales." Acta Agronómica 64 (2): 194-205. 